\title{
Mycobacterium shinjukuense sp. nov., a slowly growing, non-chromogenic species isolated from human clinical specimens
}

\author{
Correspondence \\ Hajime Saito \\ hajime.saito@kanhokyo.or.jp
}

\author{
Hajime Saito, ${ }^{1} \dagger$ Tomotada Iwamoto, ${ }^{2}$ † Kiyofumi Ohkusu, ${ }^{3}+$ Yoshihito Otsuka, ${ }^{4}$ \\ Yasushi Akiyama, ${ }^{5}$ Shigeki Sato, ${ }^{6}$ Osamu Taguchi, ${ }^{7}$ Yoshiko Sueyasu, $^{8}$ \\ Yoshiko Kawabe, ${ }^{9}$ Hisao Fujimoto, ${ }^{10}$ Takayuki Ezaki $^{3}$ and Ray Butler ${ }^{11}$ \\ ${ }^{1}$ Hiroshima Environment \& Health Association, Health Science Center, Hiroshima City, Japan \\ ${ }^{2}$ Department of Microbiology, Kobe Institute of Health, Kobe City, Japan \\ ${ }^{3}$ Department of Microbiology, Regeneration and Advanced Medical Science, Gifu University \\ Graduate School of Medicine, Gifu City, Japan \\ ${ }^{4}$ Department of Laboratory Medicine, Kameda Medical Center, Chiba City, Japan \\ ${ }^{5}$ Center for Respiratory Diseases, Hokkaido Social Insurance Hospital, Sapporo City, Japan \\ ${ }^{6}$ Department of Respiratory Medicine, Nagoya City University Hospital, Nagoya City, Japan \\ ${ }^{7}$ Department of Respiratory and Critical Care Medicine, Mie University, Graduate School of \\ Medicine, Tsu City, Japan \\ ${ }^{8}$ Department of Respiratory Disease, Chikugo City Hospital, Chikugo City, Japan \\ ${ }^{9}$ Department of Respiratory Disease, National Hospital Organization, Tokyo National Hospital, \\ Kiyose City, Japan \\ ${ }^{10}$ Kumamoto Onjaku Hospital, Misato Town, Japan \\ ${ }^{11}$ Mycobacteriology Laboratory, Division of Tuberculosis Elimination, Centers for Disease Control \\ and Prevention, Atlanta, GA, USA
}

Seven isolates of a slowly growing, non-chromogenic Mycobacterium species were obtained from sputum and bronchial lavage fluid samples from elderly patients in different regions of Japan. These isolates were distinguished from related non-tuberculous species by colony morphology, positive results for Tween hydrolysis, catalase at $68{ }^{\circ} \mathrm{C}$, nitrate reductase and pyrazinamidase and negative results for semi-quantitative catalase, urease and arylsulfatase. The mycolic acid pattern obtained by HPLC revealed a single cluster of late-eluting mycolic acids similar to but different from those of Mycobacterium malmoense ATCC $29571^{\top}$. The 16S rRNA gene, 16S-23S internal transcribed spacer (ITS), rpoB and $h s p 65$ sequences were unique in comparison with those of other mycobacteria. Comparison of 16S rRNA gene sequences showed that the isolates were most

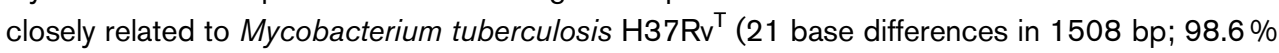
$16 \mathrm{~S}$ rRNA gene sequence similarity). A representative strain, GTC $2738^{\top}$, showed $91.9 \%$ rpoB sequence similarity with Mycobacterium marinum strain M, 95\% hsp65 sequence similarity with Mycobacterium kansasii CIP $104589^{\top}$ and $81.1 \% 16$ S-23S ITS sequence similarity with Mycobacterium gordonae ATCC $14470^{\top}$. Phylogenetic analysis of concatenated sequences of the $16 \mathrm{~S}$ rRNA, rpoB and $h s p 65$ genes showed that strain GTC $2738^{\top}$ was located on a distinct clade adjacent to $M$. tuberculosis, M. ulcerans and M. marinum, with bootstrap values of $81 \%$. DNADNA hybridization demonstrated less than $70 \%$ reassociation with type strains of genetically related species and supported the novel species status of the isolates. On the basis of this evidence,

†These authors contributed equally to this work.

Abbreviation: ITS, internal transcribed spacer.

The GenBank/EMBL/DDBJ accession numbers for the 16S rRNA gene, 16S-23S ITS, rpoB and hsp65 sequences of strain GTC $2738^{\top}$ are AB268503, AB551555, AB268504 and AB268505, respectively. 
a novel species with the name Mycobacterium shinjukuense sp. nov. is proposed. The type strain, isolated from a sputum sample, is strain GTC $2738^{\top}\left(=\mathrm{JCM} 14233^{\top}=\right.$ CCUG $\left.53584^{\top}\right)$.

Non-tuberculous mycobacteria are widely distributed in the environment and some of them are pathogenic to humans and animals (Marras \& Daley, 2002). The widespread use of sequence-based identification methods has increased our knowledge of the diversity within the genus Mycobacterium and has contributed to the discovery of novel taxa (Tortoli, 2003). Phylogenetic studies using the 16S rRNA gene, 16S-23S internal transcribed spacer (ITS), $r p o B$ and $h s p 65$ sequences are often useful to clarify whether an organism is a member of a known taxon or represents a hitherto-unknown taxon (Springer et al., 1996; Tortoli, 2003; van Ingen et al., 2009a, b). This study describes seven isolates obtained from pulmonary samples which yielded 16S rRNA gene, 16S-23S ITS, rpoB and hsp65 sequences that were unique when compared with other mycobacteria. A taxonomic examination, including DNA-DNA hybridization, revealed that these isolates belong to a novel species of the genus Mycobacterium.

Seven acid-fast isolates obtained from six sputum and one bronchial lavage fluid samples, previously identified as Mycobacterium sp., were submitted to our laboratory for species identification. These isolates were collected individually from five female and two male elderly patients, representing multiple geographical regions throughout Japan. The demographics of the isolates are summarized in Table 1. None of the patients had been diagnosed with any underlying immunocompromising disease. Of the seven cases, two (cases 1 and 2) satisfied the diagnostic criteria for pulmonary non-tuberculous mycobacterial disease proposed by the American Thoracic Society (Griffith et al., 2007) and were considered clinically relevant in the patients from whom the strains were isolated. In the other five cases, their clinical relevance was not known because of a lack of medical information.
The bacterial morphology for each isolate was determined by acid-fast staining using the Ziehl-Neelsen staining method. Colony morphology, pigment production under dark and light conditions and the ability to grow at 25$45{ }^{\circ} \mathrm{C}$ were examined on Middlebrook $7 \mathrm{H} 11$ and $2 \%$ Ogawa egg medium. The following biochemical tests were performed as described by Kent \& Kubica (1985): nitrate reductase, arylsulfatase on day 3 , semi-quantitative catalase $(45 \mathrm{~mm}), 68{ }^{\circ} \mathrm{C}$ catalase, Tween 80 hydrolysis, urease activity, tolerance of $5 \% \mathrm{NaCl}$ and pyrazinamidase. All seven isolates exhibited the same results in the above biochemical and phenotypic identification tests. We also performed the above identification tests on four closely related reference strains (Mycobacterium tuberculosis ATCC $27294^{\mathrm{T}}, M$. kansasii ATCC $12478^{\mathrm{T}}$, M. marinum ATCC $927^{\mathrm{T}}$ and M. ulcerans ATCC $19423^{\mathrm{T}}$ ). The results are given in the species description and Table 2.

Analysis of the cell-wall mycolic acid of a representative strain, GTC $2738^{\mathrm{T}}$, was performed by HPLC using methods described elsewhere (Butler \& Guthertz, 2001). Strain GTC $2738^{\mathrm{T}}$ was characterized by having a single cluster of mycolic acid peaks (Fig. 1). The pattern was compared at the Centers for Disease Control and Prevention (CDC) to those in a HPLC reference library for type strains and in an online image database at http:// www.MycobacToscana.it/. The pattern was visually similar to that of $M$. malmoense ATCC $29571^{\mathrm{T}}$; however, strain GTC $2738^{\mathrm{T}}$ produced mycolic acid peaks that eluted with a retention time, relative to the high molecular mass standard, of 3.78-0.35 min, which differed from the relative retention time of $3.50-0.70$ min for M. malmoense ATCC $29571^{\mathrm{T}}$. In addition, strain GTC $2738^{\mathrm{T}}$ demonstrated peaks with relative retention times of $0.60,0.48$ and $0.35 \mathrm{~min}$, which were absent from

Table 1. Demographics of the clinical strains

BLF, Bronchial lavage fluid; F, female; M, male; TB, tuberculosis.

\begin{tabular}{|llccllll|}
\hline Case & Strain & \multicolumn{2}{c}{ Patient } & $\begin{array}{c}\text { Year of } \\
\text { isolation }\end{array}$ & Source & Location & \multicolumn{1}{c|}{ Symptoms } \\
\cline { 3 - 5 } & & Sex & Age & & & \\
\hline 1 & GTC $2738^{\mathrm{T}}$ & F & 73 & 2004 & Sputum & Tokyo & Cavitary lesion \\
2 & UN-115 & F & 73 & 2005 & Sputum & Hokkaido & $\begin{array}{c}\text { Bronchiectasis, infiltrative shadow on middle lobe } \\
\text { and lingula }\end{array}$ \\
3 & UN-126 & M & 78 & 2005 & Sputum & Aichi & Previous history of pneumoconiosis \\
4 & UN-129 & F & 64 & 2006 & BLF & Mie & Bronchiectasis \\
5 & UN-130 & F & 89 & 2006 & Sputum & Fukuoka & Pneumonia \\
6 & UN-131 & F & 57 & 2006 & Sputum & Tokyo & Cavitary lesion \\
7 & UN-132 & M & 89 & 2006 & Sputum & Kumamoto & Cavitary lesion, previous history of TB \\
\hline
\end{tabular}


Table 2. Phenotypic characteristics of strain GTC $2738^{\top}$ and its closest phylogenetic neighbours

Strains: 1, Mycobacterium shinjukuense sp. nov. GTC $2738^{\mathrm{T}} ; 2, \mathrm{M}$. tuberculosis ATCC $27294^{\mathrm{T}} ; 3$, M. kansasii ATCC $12478^{\mathrm{T}} ; 4$, M. marinum ATCC $927^{\mathrm{T}} ; 5$, M. ulcerans ATCC $19423^{\mathrm{T}}$. All data were obtained in this study. All strains grew at $30{ }^{\circ} \mathrm{C}$, and none of the strains grew at $45{ }^{\circ} \mathrm{C}$ or with $5 \% \mathrm{NaCl}$; all strains were negative for arylsulfatase activity at 3 days.

\begin{tabular}{|lccccc|}
\hline Characteristic & $\mathbf{1}$ & $\mathbf{2}$ & $\mathbf{3}$ & $\mathbf{4}$ & $\mathbf{5}$ \\
\hline Colony morphology & $\mathrm{S}$ & $\mathrm{R}$ & $\mathrm{S} / \mathrm{R}$ & $\mathrm{S} / \mathrm{R}$ & $\mathrm{R}$ \\
Pigmentation $\dagger$ & $\mathrm{N}$ & $\mathrm{N}$ & $\mathrm{P}$ & $\mathrm{P}$ & $\mathrm{N}$ \\
Growth at: & & & & & \\
$\quad 25{ }^{\circ} \mathrm{C}$ & - & - & + & + & - \\
$37{ }^{\circ} \mathrm{C}$ & + & + & + & - & + \\
$42{ }^{\circ} \mathrm{C}$ & - & - & + & - & - \\
Tween 80 hydrolysis at 10 days & + & - & + & + & - \\
Urease & - & + & + & + & - \\
Nitrate reduction & + & + & + & - & - \\
Catalase $>45$ mm & - & - & + & - & + \\
Catalase $68{ }^{\circ} \mathrm{C}$ & + & - & + & - & + \\
Pyrazinamidase & + & + & - & + & - \\
\end{tabular}

R, Rough; s, smooth.

N, Non-chromogenic; P, photochromogenic.

M. malmoense ATCC $29571^{\mathrm{T}}$ (not shown). Visually, the patterns did not match any of the existing HPLC patterns in the CDC library database or the online image website; thus, strain GTC $2738^{\mathrm{T}}$ was considered to represent a novel species.

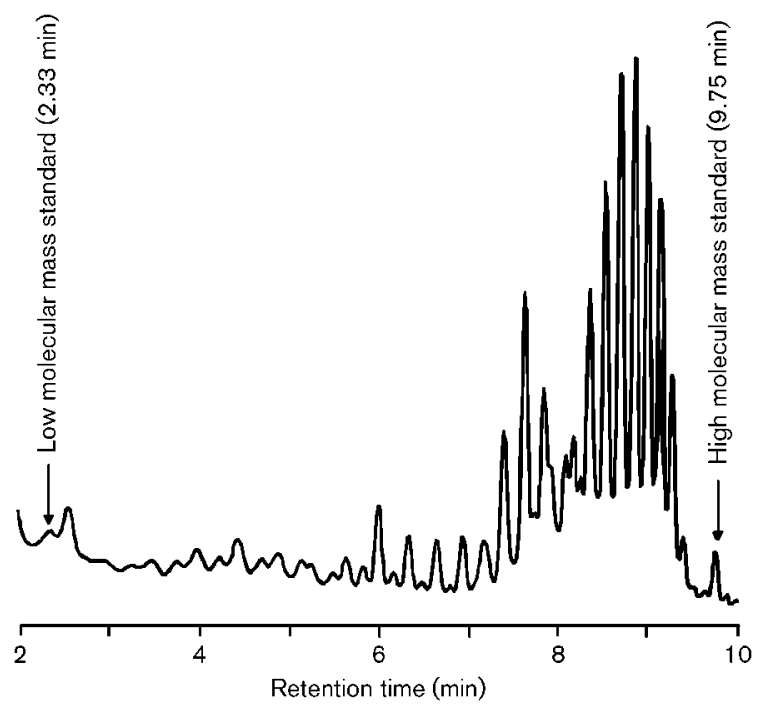

Fig. 1. Representative mycolic acid pattern of strain GTC $2738^{\top}$ obtained by HPLC analysis.
Genomic DNA was extracted from the isolates using the Isoplant kit (Nippon Gene), according to the manufacturer's instructions. An almost-complete 16S rRNA gene sequence (1508 bp), the entire 16S-23S ITS sequence (286 bp) and partial rpoB (400 bp) and hsp65 (401 bp) sequences were determined using methods described elsewhere (Telenti et al., 1993; Roth et al., 1998; Devulder et al., 2005; Nakanaga et al., 2007). The sequences corresponding to the primer binding sites were excluded manually from the data. The sequences obtained in this study were compared with sequences from GenBank using BLAST (http://blast.ncbi.nlm.nih.gov/Blast.cgi).

The seven isolates had identical 16S rRNA gene, ITS, $r p o B$ and $h s p 65$ sequences, but the sequences were different from those available in GenBank. High 16S rRNA gene sequence similarity was observed between the isolates and $M$. tuberculosis $\mathrm{H}_{37 \mathrm{Rv}^{\mathrm{T}}}$ (GenBank accession no. BX842576; $98.6 \%$ similarity across $1508 \mathrm{bp}$ ), which was possibly related to a cross-reaction between the isolates and commercial tuberculosis identification kits, as reported for Mycobacterium celatum (Somoskövi et al., 2000) and Mycobacterium riyadhense (van Ingen et al., 2009a). Aono et al. (2010) confirmed that the isolates gave positive cross-reactions for the TRC Rapid M.TB assay (Tosoh Bioscience) and AMPLIFIED MTD Test (Gen-Probe), while the COBAS AMPLICOR MTB (Roche Diagnostics), COBAS TaqMan MTB (Roche Diagnostics) and AccuProbe MTB (Gen-Probe) assays showed negative reactions. Strain GTC $2738^{\mathrm{T}}$ exhibited the highest $r p o B$ sequence similarities with $M$. marinum strain M (CP000854; $91.9 \% \quad r p o B$ sequence similarity across $395 \mathrm{bp}$ ) and M. ulcerans Agy99 (CP000325; $91.7 \%$ across $399 \mathrm{bp}$ ) and the highest $h s p 65$ sequence similarity with $M$. kansasii CIP $104589^{\mathrm{T}}$ ( $95.0 \%$ hsp65 sequence similarity across $401 \mathrm{bp}$ ). The highest ITS sequence similarity was observed between strain GTC $2738^{\mathrm{T}}$ and Mycobacterium gordonae ATCC $14470^{\mathrm{T}}$ (GenBank accession no. L42260); however, this similarity was low, with 230 matches out of $283 \mathrm{bp}(81.1 \%)$.

In an attempt to clarify the phylogenetic position of the isolates within the slowly growing mycobacteria, a concatenated phylogenetic tree using $16 \mathrm{~S}$ rRNA gene (1341 bp), rpoB (398 bp) and hsp65 (401 bp) sequences was constructed. The ITS sequence was not included in the analysis as the sequence is too variable to construct reliable trees. Phylogenetic trees were generated using CLUSTAL W version 1.83 (Thompson et al., 1994) and displayed using TreeView (Page, 1996). Analyses were performed using the neighbour-joining method with Kimura's two-parameter distance model and 1000 bootstrap replications. Phylogenetic analysis of the 16S rRNA gene sequence (Fig. 2a) showed that strain GTC $2738^{\mathrm{T}}$ was located in a clade adjacent to M. tuberculosis NCTC $7416^{\mathrm{T}}$, M. ulcerans Borstel $10564 / 70$ and M. marinum DSM $44344^{\mathrm{T}}$, with $79.9 \%$ bootstrap support. The bootstrap values in the phylogenetic trees of the $r p o B$ (Fig. 2b) and $h s p 65$ (Fig. 2c) sequences were too low to indicate the phylogenetic position of the isolate. Analysis of the concatenated sequences (Fig. 2d) confirmed that strain GTC $2738^{\mathrm{T}}$ formed a separate clade within the 
(a) $16 \mathrm{~S}$ rRNA gene

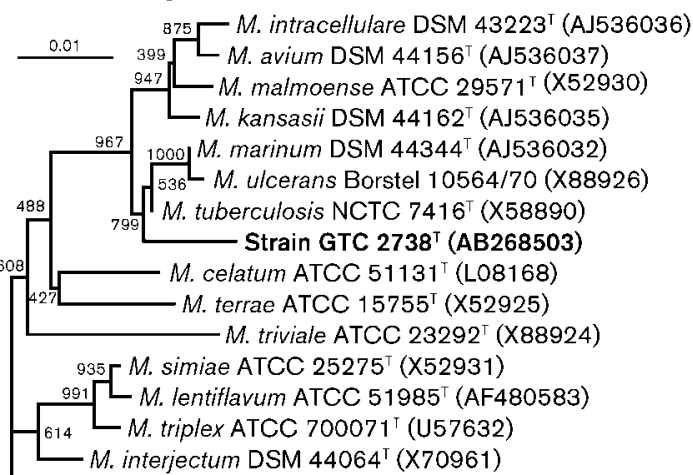

Nocardia asteroides DSM 43757 (AF430019)

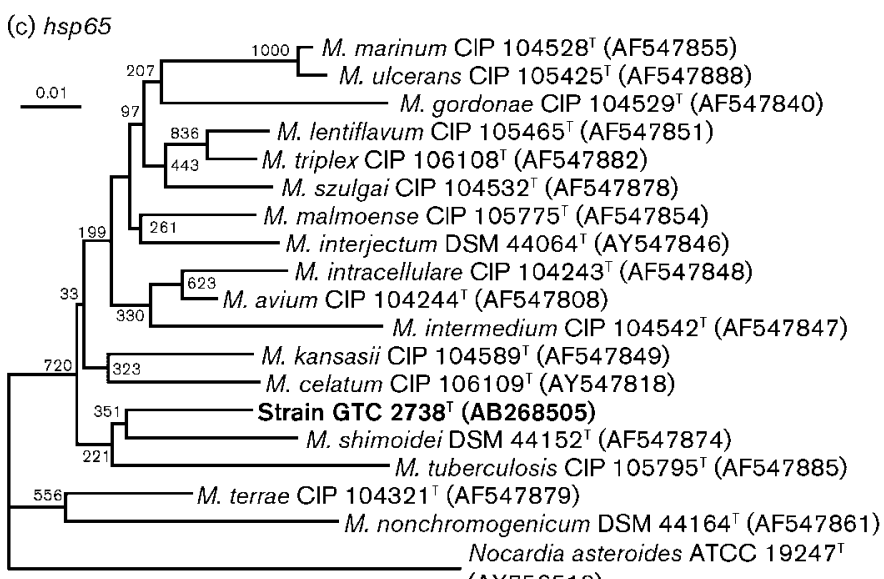
(AY756513)

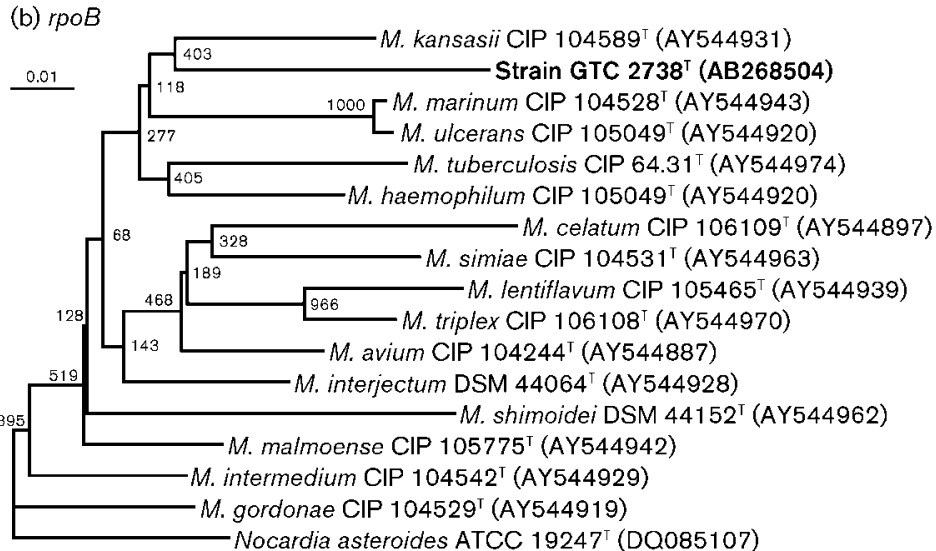

(d) Concatenation of $16 \mathrm{~S}$ rRNA, rpoB and $h s p 65$ genes

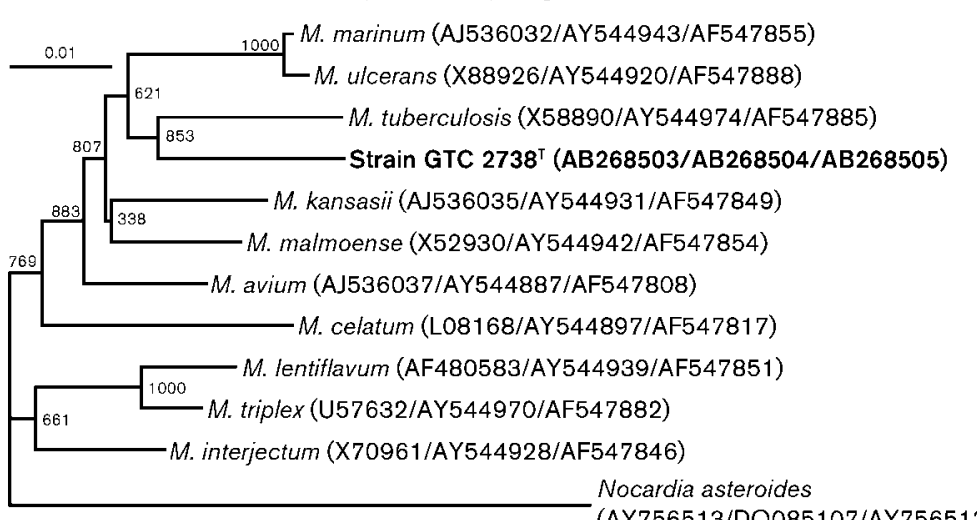

Fig. 2. Neighbour-joining phylogenetic trees showing the positions of strain GTC $2738^{\top}$ and closely related type strains of $M y c o b a c t e r i u m$ species based upon comparison of sequences of $16 \mathrm{~S}$ rRNA gene (a), rpoB (b) and $h s p 65$ (c) and a concatenation of $16 \mathrm{~S}$ rRNA gene, rpoB and hsp65 sequences (d). Bootstrap values ( $>500$ ) based on 1000 replications are shown at branch nodes. Nocardia asteroides ATCC $19247^{\top}$ was used as an outgroup. Bars, $1 \%$ sequence divergence. 
Table 3. DNA-DNA relatedness between strain GTC $2738^{\top}$ and members of the genus Mycobacterium

\begin{tabular}{|c|c|c|c|}
\hline \multirow[t]{2}{*}{ Strain } & \multicolumn{3}{|c|}{$\begin{array}{l}\text { DNA-DNA hybridization } \\
\text { (\%) with labelled DNA from }\end{array}$} \\
\hline & 1 & 2 & 3 \\
\hline 1. Strain GTC $2738^{\mathrm{T}}$ & 100 & 30 & 30 \\
\hline 2. M. tuberculosis ATCC $27294^{\mathrm{T}}$ & 40 & 100 & ND \\
\hline 3. M. marinum ATCC $927^{\mathrm{T}}$ & 20 & ND & 100 \\
\hline M. bovis ATCC $19210^{\mathrm{T}}$ & 61 & 111 & ND \\
\hline M. microti ATCC $19422^{\mathrm{T}}$ & 42 & 83 & ND \\
\hline M. ulcerans ATCC $19423^{\mathrm{T}}$ & 21 & ND & 81 \\
\hline
\end{tabular}

ND, Not done.

cluster containing the type strains of $M$. tuberculosis, $M$. ulcerans and M. marinum.

Given the close phylogenetic position of strain GTC $2738^{\mathrm{T}}$ to the M. tuberculosis complex, M. marinum and M. ulcerans, quantitative microplate DNA-DNA hybridization was carried out under optimal conditions as described by Ezaki et al. $(1988,1989)$. Strain GTC $2738^{\mathrm{T}}$ exhibited $<70 \%$ DNA-DNA relatedness with members of the genus Mycobacterium (Table 3), below the suggested threshold for the delineation of species. This result provided further evidence of genetic divergence between strain GTC $2738^{\mathrm{T}}$ and its closest phylogenetic neighbours.

On the basis of phenotypic, chemotaxonomic and phylogenetic analysis, strain GTC $2738^{\mathrm{T}}$ is considered to represent a novel species in the genus Mycobacterium, for which the name Mycobacterium shinjukuense sp. nov. is proposed.

\section{Description of Mycobacterium shinjukuense sp. nov.}

Mycobacterium shinjukuense (shin.ju'ku.en'se. N.L. neut. adj. shinjukuense pertaining to Shinjuku ward, Tokyo, Japan, where the type strain was isolated).

Acid-alcohol-fast, rod-shaped bacilli. Grows to maturity in 21 days at $30-37{ }^{\circ} \mathrm{C}$. Colonies on $2 \%$ Ogawa egg medium are smooth and non-chromogenic. Positive in tests for Tween 80 hydrolysis, $68{ }^{\circ} \mathrm{C}$ catalase, nitrate reductase and pyrazinamidase. Negative in tests for semi-quantitative catalase, urease and 3-day arylsulfatase. Does not grow with $5 \% \mathrm{NaCl}$. Identifiable by unique $16 \mathrm{~S}$ rRNA gene, 16S-23S ITS, $h s p 65$ and $r p o B$ sequences.

The type strain is GTC $2738^{\mathrm{T}}\left(=\mathrm{JCM} 14233^{\mathrm{T}}=\mathrm{CCUG}\right.$ $\left.53584^{\mathrm{T}}\right)$, recovered from a sputum specimen.

\section{Acknowledgements}

This work was partly supported by grants from JSPS Grant-in-Aid for Scientific Research (A) (grant no. 20249007) and the United StatesJapan Cooperative Medical Science Program (TB and Leprosy panel).

\section{References}

Aono, A., Kazumi, Y., Maeda, S., Azuma, Y., Tsuchiya, S., Iwamoto, T., Nakanaga, K., Hayakawa, K. \& Saito, H. (2010). [Non-tuberculous Mycobacterium strains that show positive test for identification kits of $M$. tuberculosis complex]. Kekkaku 85, 461-464 (in Japanese).

Butler, W. R. \& Guthertz, L. S. (2001). Mycolic acid analysis by highperformance liquid chromatography for identification of Mycobacterium species. Clin Microbiol Rev 14, 704-726.

Devulder, G., Pérouse de Montclos, M. \& Flandrois, J. P. (2005). A multigene approach to phylogenetic analysis using the genus Mycobacterium as a model. Int J Syst Evol Microbiol 55, 293-302.

Ezaki, T., Hashimoto, Y., Takeuchi, N., Yamamoto, H., Liu, S.-L., Miura, H., Matsui, K. \& Yabuuchi, E. (1988). Simple genetic method to identify viridans group streptococci by colorimetric dot hybridization and fluorometric hybridization in microdilution wells. J Clin Microbiol 26, 1708-1713.

Ezaki, T., Hashimoto, Y. \& Yabuuchi, E. (1989). Fluorometric deoxyribonucleic acid-deoxyribonucleic acid hybridization in microdilution wells as an alternative to membrane filter hybridization in which radioisotopes are used to determine genetic relatedness among bacterial strains. Int $J$ Syst Bacteriol 39, 224-229.

Griffith, D. E., Aksamit, T., Brown-Elliott, B. A., Catanzaro, A., Daley, C., Gordin, F., Holland, S. M., Horsburgh, R., Huitt, G. \& other authors (2007). An official ATS/IDSA statement: diagnosis, treatment, and prevention of nontuberculous mycobacterial diseases. Am J Respir Crit Care Med 175, 367-416.

Kent, P. T. \& Kubica, G. P. (1985). Public Health Mycobacteriology. A Guide for the Level III Laboratory. Atlanta, GA: Department of Health and Human Services.

Marras, T. K. \& Daley, C. L. (2002). Epidemiology of human pulmonary infection with nontuberculous mycobacteria. Clin Chest Med 23, 553-567.

Nakanaga, K., Ishii, N., Suzuki, K., Tanigawa, K., Goto, M., Okabe, T., Imada, H., Kodama, A., Iwamoto, T. \& other authors (2007). 'Mycobacterium ulcerans subsp. shinshuense' isolated from a skin ulcer lesion: identification based on $16 \mathrm{~S}$ rRNA gene sequencing. J Clin Microbiol 45, 3840-3843.

Page, R. D. (1996). TreeView: an application to display phylogenetic trees on personal computers. Comput Appl Biosci 12, 357-358.

Roth, A., Fischer, M., Hamid, M. E., Michalke, S., Ludwig, W. \& Mauch, H. (1998). Differentiation of phylogenetically related slowly growing mycobacteria based on 16S-23S rRNA gene internal transcribed spacer sequences. J Clin Microbiol 36, 139-147.

Somoskövi, A., Hotaling, J. E., Fitzgerald, M., Jonas, V., Stasik, D., Parsons, L. M. \& Salfinger, M. (2000). False-positive results for Mycobacterium celatum with the AccuProbe Mycobacterium tuberculosis complex assay. J Clin Microbiol 38, 2743-2745.

Springer, B., Stockman, L., Teschner, K., Roberts, G. D. \& Böttger, E. C. (1996). Two-laboratory collaborative study on identification of mycobacteria: molecular versus phenotypic methods. J Clin Microbiol 34, 296-303.

Telenti, A., Marchesi, F., Balz, M., Bally, F., Böttger, E. C. \& Bodmer, T. (1993). Rapid identification of mycobacteria to the species level by polymerase chain reaction and restriction enzyme analysis. J Clin Microbiol 31, 175-178.

Thompson, J. D., Higgins, D. G. \& Gibson, T. J. (1994). CLUSTAL W: improving the sensitivity of progressive multiple sequence alignment through sequence weighting, position-specific gap penalties and weight matrix choice. Nucleic Acids Res 22, 4673-4680. 
Tortoli, E. (2003). Impact of genotypic studies on mycobacterial taxonomy: the new mycobacteria of the 1990s. Clin Microbiol Rev 16, 319-354.

van Ingen, J., Al-Hajoj, S. A., Boeree, M., Al-Rabiah, F., Enaimi, M., de Zwaan, R., Tortoli, E., Dekhuijzen, R. \& van Soolingen, D. (2009a). Mycobacterium riyadhense sp. nov., a non-tuberculous species identified as Mycobacterium tuberculosis complex by a commercial line-probe assay. Int J Syst Evol Microbiol 59, 1049-1053.

van Ingen, J., Lindeboom, J. A., Hartwig, N. G., de Zwaan, R., Tortoli, E., Dekhuijzen, P. N., Boeree, M. J. \& van Soolingen, D. (2009b). Mycobacterium mantenii sp. nov., a pathogenic, slowly growing, scotochromogenic species. Int J Syst Evol Microbiol 59, 2782-2787. 